\title{
Mobility Increases the Connectivity of K-hop Clustered Wireless Networks
}

\author{
Qingsi Wang \\ Dept. of Elec. Engin. \\ Shanghai JiaoTong Univ. \\ Shanghai, China \\ wqswolf@sjtu.edu.cn
}

\author{
Xinbing Wang \\ Dept. of Elec. Engin. \\ Shanghai JiaoTong Univ. \\ Shanghai, China \\ xwang8@sjtu.edu.cn
}

\author{
Xiaojun Lin \\ Dept. of Elec. and Comp. \\ Engin. \\ Purdue University, USA \\ linx@ecn.purdue.edu
}

\begin{abstract}
In this paper we investigate the connectivity for large-scale clustered wireless sensor and ad hoc networks. We study the effect of mobility on the critical transmission range for asymptotic connectivity in $k$-hop clustered networks, and compare to existing results on non-clustered stationary networks. By introducing $k$-hop clustering, any packet from a cluster member can reach a cluster head within $k$ hops, and thus the transmission delay is bounded as $\Theta(1)$ for any finite $k$. We first characterize the critical transmission range for connectivity in mobile $k$-hop clustered networks where all nodes move under either the random walk mobility model with non-trivial velocity or the i.i.d. mobility model. By the term non-trivial velocity, we mean that the velocity of nodes $v$ is $\Theta(1)$. We then compare with the critical transmission range for stationary $k$-hop clustered networks. We also study the transmission power versus delay trade-off and the average energy consumption per flow among different types of networks. We show that random walk mobility with nontrivial velocity increases connectivity in $k$-hop clustered networks, and thus significantly decreases the energy consumption and improves the power-delay trade-off. The decrease of energy consumption per flow is shown to be $\Theta\left(\frac{\log n}{n^{d}}\right)$ in clustered networks. These results provide insights on network design and fundamental guidelines on building a large-scale wireless network.
\end{abstract}

\section{Categories and Subject Descriptors}

C.2.1 [Computer-Communication Networks]: Network Architecture and Design-Wireless Communications

\section{General Terms}

Theory and Algorithms

\section{Keywords}

Connectivity, Clustered Networks, Mobility, Scaling Law

Permission to make digital or hard copies of all or part of this work for personal or classroom use is granted without fee provided that copies are not made or distributed for profit or commercial advantage and that copies bear this notice and the full citation on the first page. To copy otherwise, to republish, to post on servers or to redistribute to lists, requires prior specific permission and/or a fee.

MobiCom'09, September 20-25, 2009, Beijing, China.

Copyright 2009 ACM 978-1-60558-702-8/09/09 ...\$5.00.

\section{INTRODUCTION}

Connectivity is a basic concern in designing and implementing wireless networks, and hence is also of paramount significance. Nodes in the networks need to connect to others by adjusting their transmission power and thus carry out the network's functionalities. Therefore, three main schemes of connecting strategies are proposed in literature.

The first type of connecting strategies is distance-based. That is, for a graph (network) $G(V, E)$ and any two nodes $i, j \in V, e_{i j} \in E$ if and only if the Euclidean distance between $i$ and $j$ is at most $r$. The critical value of $r$ for connectivity when the number of nodes grows to infinity has been studied. In [1], Gupta and Kumar proved that with range $r(n)=\sqrt{\frac{\log n+c(n)}{\pi n}}$, overall connectivity can be established with probability one as $n \rightarrow \infty$ if and only if $c(n) \rightarrow \infty$. And this result was independently determined by Penrose [2] as well. In [3], Wan and Yi determined the precise critical transmission range for $k$-connectivity.

Of equal importance, the second type of connecting strategies is the number-of-neighbor-based strategy, which means that for $G(V, E)$ and any two nodes $i, j \in V, e_{i j} \in E$ if and only if $j$ is among $i$ 's $\phi$ nearest neighbors. Note that this strategy does not ensure that the degree of each node is strictly equal to $\phi$. Actually, we have the degree of each node $D_{i} \geq \phi$, since the $\phi$-nearest-neighbor relation is asymmetric. In [4], Xue and Kumar proved that for a network with $n$ nodes to be asymptotically connected, $\Theta(\log n)^{1}$ neighbors are necessary and sufficient. Wan and Yi [3] obtained an improved asymptotic upper bound on the critical neighbor number for $k$-connectivity.

Another strategy is the sector-based strategy that was proposed as a topology control algorithm by Wattenhofer et al. [5] and was further discussed by Li et al. in [6]. This strategy is based on the neighbor connection as described above and further concerns with the $\theta$-coverage problem. Given that a node connects bidirectionally to its $\phi_{n}$ nearest neighbors in the network, where $\phi_{n}$ is a deterministic function of $n$ to be specified, for an angle $\theta \in(0,2 \pi)$, the node is called to be $\theta$-covered by its $\phi_{n}$ nearest neighbors if

\footnotetext{
${ }^{1}$ The following asymptotic notations are used throughout this paper. Given non-negative functions $f(n)$ and $g(n)$ :

1. $f(n)=\Theta(g(n))$ means for two constants $0<c_{1}<c_{2}$, $c_{1} g(n) \leq f(n) \leq c_{2} g(n)$ for sufficiently large $n$.

2. $f(n) \sim g(n)$ means $\lim _{n \rightarrow \infty} \frac{f(n)}{g(n)}=1$.

3. $f(n)=o(g(n))$ means $\lim _{n \rightarrow \infty} \frac{f(n)}{g(n)}=0$.
} 
among them, it can find a node in every sector of angle $\theta$. If every node in the graph satisfies this property, the graph is called $\theta$-covered. One then wants to find the relation between $\theta$-coverage and overall connectivity of the network, and to determine the critical value of $\phi_{\theta}$ which is a deterministic function of $\theta$. In [7], Xue and Kumar determined that the exact threshold function for $\theta$-coverage, including even the pre-constant, is $\log _{\frac{2 \pi}{2 \pi-\theta}} n$, for any $\theta \in(0,2 \pi)$, and $\pi$-coverage with high probability implies overall connectivity with high probability.

The network models studied in these prior works are nonclustered (or flat) and stationary networks. Flat networks are found to have poor scalability [8] [9] and energy inefficiency [10] [11]. Clustering and mobility have been found to improve various aspects of network performance. First, clustered networks and clustering algorithms are studied by many researchers [12] [13] [14] [15] and have applications in both sensor networks [10] [16] and ad hoc networks [17] [18]. With random infrastructure support, the throughput capacity of random ad hoc networks can be greatly improved, and the capacity gain is found as $\Theta\left(\sqrt{\frac{n}{\log n}}\right)$ when the number of ad hoc nodes per access point is bounded as $\Theta(1)$ [19]. In [10], Heinzelman et al. presented that in sensor networks where nodes have sinks or base stations to gather their data, organizing nodes into clusters and using cluster head electing and rotating can be more energy-efficient than non-clustered multi-hop transmission to base stations which is normally adopted in ad hoc networks. In a separate direction, mobility has been found to increase the capacity [20] and help security [21] in ad hoc networks.

However, compared to the relatively mature study on the connectivity of flat and stationary networks, studies on the connectivity of mobile and clustered networks are quite limited. In a clustered network, a packet only needs to reach one of the cluster heads. We are interested in two cases in this paper. In a stationary $k$-hop clustered network, a packet must reach a cluster head within $k$ hops. In a mobile $k$-hop clustered network, a packet must reach a cluster head directly in $k$ time-slots. Clearly, clustering has an inherent advantage compared to flat networks, and it can alter the energy efficiency and delay of the system. First, it can require a different critical transmission range for connectivity, which may depend on the number of cluster heads and whether the network is stationary or mobile. Second, it can lead to different delay. For example, with $k$-hop clustering, the delay is bounded by $k$ (i.e., $\Theta(1)$ ). In contrast, in a flat network with the minimum transmission range, the number of hops will increase as $\Theta\left(\sqrt{\frac{n}{\log n}}\right)$, and so does the delay. Finally, both the transmission range and the number of hops can affect the energy consumption of the network. We can then ask the following open question in this paper:

- What is the impact of mobility on connectivity of clustered networks subject to delay constraints?

In this paper, we concentrate on one of the above connecting strategies, namely, the distance-based strategies. We study the critical transmission range for connectivity in mobile $k$-hop clustered networks where all nodes move under either the random walk mobility model with non-trivial velocity or the i.i.d. mobility model. By the term non-trivial velocity, we mean that the velocity of nodes $v$ is $\Theta(1)$. We then compare with the critical transmission range for con- nectivity in stationary $k$-hop clustered networks. We also use these results to study the power-delay trade-off and the energy efficiency of different types of networks, including flat networks. Our results show that random walk mobility with non-trivial velocity does improve connectivity in $k$-hop clustered networks, and it also significantly decreases the energy consumption and the power-delay trade-off. Hence, these results provide fundamental insights on the design of large-scale wireless networks.

The rest of the paper is organized as follows. In section 2 , we describe the $k$-hop clustered network models. We provide the main results and some intuition behind these results in section 3 . In section 4,5 and 6 , we give the proofs of the critical transmission range in mobile $k$-hop clustered networks under the random walk mobility model with non-trivial velocity and the i.i.d. mobility model, and in stationary $k$-hop clustered networks, respectively. Then we have a discussion on the impact of mobility on connectivity and network performance in $k$-hop clustered networks in section 7 . We conclude in section 8 .

\section{K-HOP CLUSTERED NETWORK MOD- ELS}

In this section, we first provide an overview of flat networks and then introduce models of clustered networks. A classification of $k$-hop clustered networks is given and related issues such as the transmission scheme and the routing strategy are presented, respectively.

\subsection{An overview of flat networks}

Before studying clustered networks, we now give an overview of the so-called flat networks as depicted in Figure 1. A flat network can be defined as a network in which all nodes have homogeneous roles and functionalities (while they may have different hardware capabilities), and they can reach each other without going through any intermediary service points such as base stations or sinks. In one word, flat networks are self-organized and infrastructure-free, like ad hoc networks in common context.

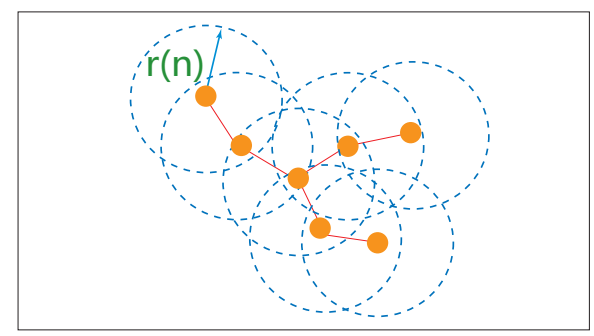

Figure 1: Flat networks under the distance-based connecting strategies

There are several concepts related to flat networks whose counterparts in clustered networks will be studied in the rest of this paper. The most concerned in this paper is connectivity. Before defining connectivity of flat networks, we formulate flat networks as follows. Let $\mathcal{A}$ denote a unit area in $\mathfrak{R}^{2}$, and $\mathcal{G}(n)$ be the graph (network) formed when $n$ nodes are placed uniformly and independently in $\mathcal{A}$. An edge $e_{i j}$ exists between two nodes $i$ and $j$, if the distance between them is less than $r(n)$ under the distance-based 
strategy. Then, graph $\mathcal{G}(n)$ is connected if and only if there is a path between any pair of nodes in $\mathcal{G}(n)$.

\subsection{Classification of k-hop clustered networks}

In contrast to flat networks, in clustered networks nodes are organized into clusters. A cluster head is selected within each cluster to serve the other cluster-member nodes (i.e., clients).

We assume that a clustered network consists of $n$ clustermember nodes and $n^{d}$ cluster-head nodes, where $d$ is called the cluster head exponent and $0<d \leq 1$. For ease of presentation, we treat $n^{d}$ as an integer in this paper, and all nodes are placed uniformly and independently in a unit square $\mathcal{S}$ in $\mathfrak{R}^{2}$. Moreover, the unit square is assumed to be a torus.

\subsubsection{Mobile k-hop clustered networks}

a. Mobility pattern

In a mobile $k$-hop clustered network, we assume that all cluster members move according to a certain mobility pattern while the clustered heads are fixed with the uniform distribution.

- Random Walk Mobility Model with Non-Trivial Velocity $^{2}$ [22]: We partition the data transmission process into time-slots with unit length. At the beginning of each time-slot each member node will uniformly choose a random direction $\theta \in[0,2 \pi)$. The node moves along this direction with a constant velocity $v$ for each time-slot and the velocity $v$ is $\Theta(1)$. Note that the mobility pattern of nodes in our model is slightly different from that defined in [22] and they do not bounce off the border since we have assumed the unit square to be a torus.

- I.I.D. Mobility Model: The transmission process is also divided into slots as we did above and at the beginning of each time-slot each member node will randomly and uniformly choose a position within the unit torus and remain static during the rest of the time-slot. Besides, we assume that $d>\frac{1}{k}$. (Please refer to the proof of Proposition 5.1 where we need the condition that $d>\frac{1}{k}$.)

Due to the assumption that $v=\Theta(1)$, the mixing time under the r.w. mobility model is on the same order as the mixing time under the i.i.d. mobility model. However, since under the r.w. mobility model nodes can communicate during the course of movement, they will be a higher chance to connect to the cluster head compared to nodes under the i.i.d. mobility model. This advantage will become clear after we define the transmission scheme.

b. Transmission scheme

We divide the channel into $W(W \geq 2)$ sub-channels, and thus the network can accommodate at least $W$ flows initiated in a certain time-slot. Moreover, we assume that for each flow, the packet is forwarded for one hop in each timeslot. Therefore, the maximum delay for the transmission of a packet in our network model is $k$ time-slots, or the delay constraint is $D=k$. In section 7 , we will mainly use the

\footnotetext{
${ }^{2}$ In the random walk mobility model defined in [22], each movement either corresponds to a constant time interval $t$, or corresponds to a constant distance traveled. The model we use conforms with the former case.
}

notation $D$ as the delay constraint in our discussion on the power-delay trade-off in $k$-hop clustered networks.

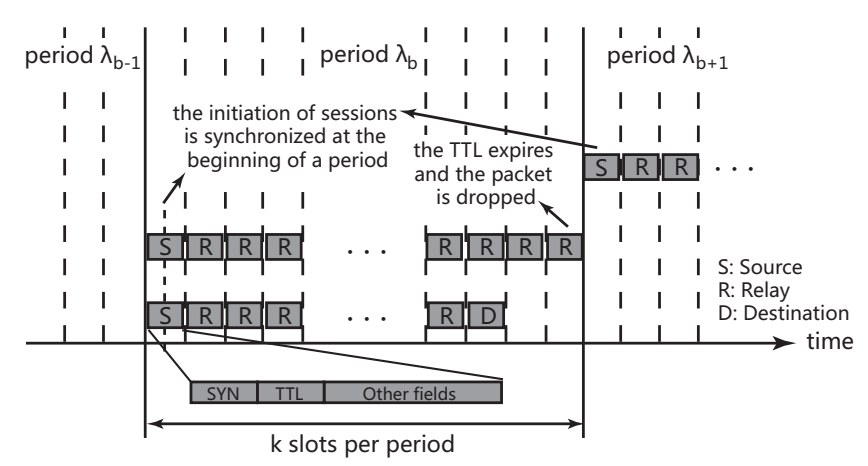

Figure 2: Transmission scheme in mobile $k$-hop clustered networks.

We use the term session to refer to the process that a packet is forwarded from its source cluster member to a cluster head. In every packet, we assume that there is a TTL (time to live) field to record the number of hops that the packet has been forwarded. The initial value of TTL is set to 1 and each relay increases the counter by one when it receives the packet. When the hop counter is greater than $k$, the packet is discarded and we say that the session is failed. Every $k$ time-slots constitute a period. We assume that there is a SYN (synchronize) field for all nodes to be synchronized and data-flows are initiated only at the beginning of each period. This assumption accords with the design of some novel energy-efficient duty-circle MAC protocols (RMAC [23], DW-MAC [24]). Our proposed transmission scheme is illustrated in Figure 2.

\section{c. Routing strategy}

As to the routing strategy, we simply assume that a cluster member holds the packet (acting as the relay of itself), if it does not have a cluster head in its transmission range during its course of movement, or sends the packet to the cluster head once they meet.

Note that this assumption requires that a cluster member can know the existence of a cluster head within the transmission range. Such an assumption would be valid when (1) the cluster heads are static and the cluster member has knowledge of its own position and the positions of cluster heads; or (2) the cluster heads broadcast a pilot signal that covers nearby cluster members. Our routing strategy under the random walk mobility assumption is illustrated in Figure 3.

Currently, we do not actually use multi-hop transmissions in mobile $k$-hop clustered networks because multi-hop transmissions require significantly higher overhead due to the need to discover cluster heads at a further distance away and to establish multi-hop paths on demand. Thus, we proposed a simplified routing strategy to avoid the technicalities of a more complicated one which may obscure our main target.

\subsubsection{Stationary $k$-hop clustered networks}

In a stationary $k$-hop clustered network, all nodes remain static after uniformly distributed in the unit area. As in its mobile counterpart, we also assume that the packet is forwarded for one hop in each time-slot. 


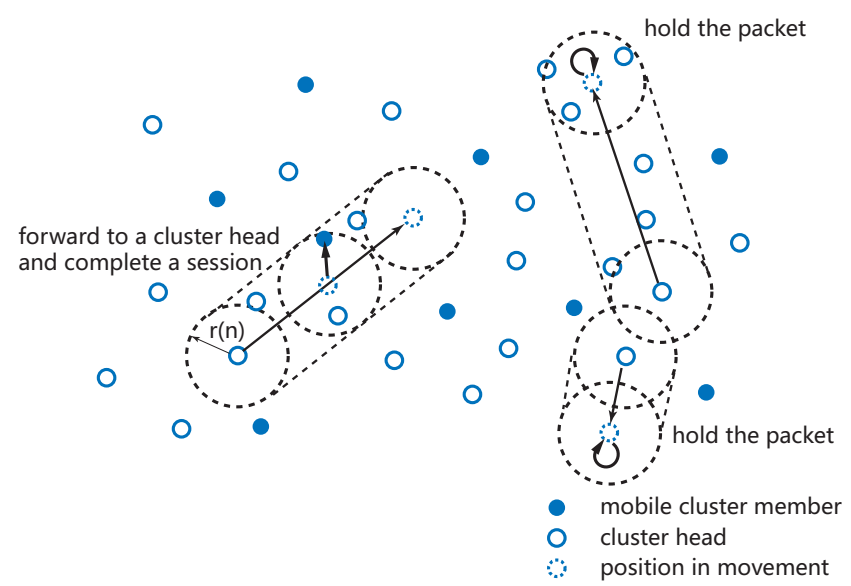

Figure 3: Routing strategy in mobile $k$-hop clustered networks, random walk mobility.

\subsubsection{Redefining connectivity in clustered networks}

Due to clustering and mobility, the definition of connectivity in clustered networks is different from that in flat networks. For stationary $k$-hop clustered networks, we say that a cluster member is connected if it can reach a cluster head within $k$ hops. For mobile clustered networks, a cluster member is connected if it can reach a cluster head within $k$ slots. If all the cluster members in a network are connected, we define that the network has full connectivity.

\section{MAIN RESULTS AND INTUITIONS}

Before we state our main results, we first formally define the critical transmission range in both mobile and stationary $k$-hop clustered networks.

For stationary or mobile $k$-hop clustered networks, let $E$ denote the event that all cluster members are connected.

Definition 3.1. For stationary or mobile $k$-hop clustered networks, $r(n)$ is the critical transmission range if

$$
\begin{aligned}
& \lim _{n \rightarrow \infty} P(\{E\})=1, \text { if } r \geq c r(n) \text { and } c>1 ; \\
& \lim _{n \rightarrow \infty} P(\{E\})<1, \text { if } r \leq c^{\prime} r(n) \text { and } c^{\prime}<1 .
\end{aligned}
$$

We summarize our main results in this paper as follows:

- Under the random walk mobility assumption, the critical transmission range is $r(n)=\frac{\log n}{2 k v n^{d}}$, where $d$ is the cluster head exponent, $0<d \leq 1, v$ is the velocity of all member nodes.

- Under the i.i.d. mobility assumption, the critical transmission range is $r(n)=\sqrt{\frac{\log n}{k \pi n^{d}}}$, where $\frac{1}{k}<d \leq 1$.

- For stationary $k$-hop clustered networks, the critical transmission range is $r(n)=\frac{1}{k} \sqrt{\frac{d \log n}{\pi n^{d}}}$, where $0<$ $d<1$.

Before we prove these results rigorously, we now give an intuitive approach to estimate the order of critical transmission range in each scenario here:

Suppose there are $n$ cluster members and $n^{d}$ cluster heads uniformly distributed in a unit square. Thus, roughly speaking, there is one cluster head within an area of $\frac{1}{n^{d}}$.
- Under the random walk mobility assumption, the area covered by movement during $k$ time-slots constitutes the dominating part of a cluster member's coverage area. Thus, in order to reach a cluster head, on average we need

$$
k v r(n)=\frac{1}{n^{d}}, \text { or } r(n)=\frac{1}{k v n^{d}} .
$$

- Under the i.i.d. mobility assumption, considering that cluster members actually remain static during any timeslot, the coverage consists of the overall area of $k$ disks. Thus, on average we need

$$
k r^{2}(n)=\frac{1}{n^{d}}, \text { or } r(n)=\sqrt{\frac{1}{k n^{d}}} .
$$

- In the stationary networks, a reachable cluster head is roughly within a disk with a radius $k r(n)$ of the cluster member, and thus we need

$$
(k r(n))^{2}=\frac{1}{n^{d}}, \text { or } r(n)=\frac{1}{k} \sqrt{\frac{1}{n^{d}}} .
$$

\section{THE CRITICAL TRANSMISSION RANGE FOR MOBILE K-HOP CLUSTERED NET- WORKS, RANDOM WALK MOBILITY}

In this section, we have the following main result.

THEOREM 4.1. Under the random walk mobility assumption, the critical transmission range is $r(n)=\frac{\log n}{2 k v n^{d}}$, where $v$ is the velocity of all member nodes and $0<d \leq 1$.

\subsection{Necessary condition on $r(n)$ of Theorem 4.1}

Let $\mathcal{G}_{r w}(n, r(n))$ denote the network where two nodes can communicate if their Euclidean distance is at most $r(n)$ and $P_{f_{-} r w}(n, r(n))$ be the probability that $\mathcal{G}_{r w}(n, r(n))$ has some node that is not connected. Then we have the following proposition.

Proposition 4.1. If $r(n)=\frac{\log n+\omega(n)}{2 k v n^{d}}$, then

$$
\liminf _{n \rightarrow \infty} P_{f_{-} r w}(n, r(n)) \geq e^{-\omega}\left(1-e^{-\omega}\right),
$$

where $\omega=\lim _{n \rightarrow \infty} \omega(n)$.

ProOF. We first study the case where $r(n)=\frac{\log n+\omega}{2 k v n^{d}}$ for a fixed $\omega$. Let $s_{i}$ be the session initiated by the cluster-member node $i$. We say that the session fails if $i$ is not connected (i.e., it cannot reach a cluster head in $k$ time-slots). Then we have

$$
\begin{aligned}
& P_{f_{-} r w}(n, r(n)) \\
& \geq \sum_{i=1}^{n} P\left(\left\{s_{i} \text { is the only failed session in } \mathcal{G}_{r w}(n, r(n))\right\}\right) \\
& \geq \sum_{i=1}^{n}\left(P\left(\left\{s_{i} \text { is a failed session in } \mathcal{G}_{r w}(n, r(n))\right\}\right)\right. \\
& \quad-\sum_{j \neq i} P\left(\left\{s_{i} \text { and } s_{j}\right.\right. \text { are failed sessions } \\
& \left.\left.\left.\quad \text { in } \mathcal{G}_{r w}(n, r(n))\right\}\right)\right)
\end{aligned}
$$




$$
\begin{gathered}
\geq \sum_{i=1}^{n} P\left(\left\{s_{i} \text { is a failed session in } \mathcal{G}_{r w}(n, r(n))\right\}\right) \\
-\sum_{i=1}^{n} \sum_{j \neq i} P\left(\left\{s_{i} \text { and } s_{j}\right.\right. \text { are failed sessions } \\
\left.\left.\quad \text { in } \mathcal{G}_{r w}(n, r(n))\right\}\right) .
\end{gathered}
$$

Then we evaluate the two terms on the right hand side of (1), respectively. For the first term, we have

$$
\begin{aligned}
& P\left(\left\{s_{i} \text { is a failed session in } \mathcal{G}_{r w} \text { in period } \lambda_{b}\right\}\right) \\
& \quad \geq\left(1-\left(\pi r^{2}(n)+2 r(n) k v T\right)\right)^{n^{d}} \\
& \quad \geq(1-(1+\epsilon) 2 k v r(n))^{n^{d}}
\end{aligned}
$$

for all $n>N(\epsilon)$, where $T=1$ is the length of a time-slot and $\epsilon=o(r(n))$. As to the second term, we have

$$
\begin{aligned}
& P\left(\left\{s_{i} \text { and } s_{j} \text { are failed sessions in } \mathcal{G}_{r w} \text { in period } \lambda_{b}\right\}\right) \\
& \leq \quad 4 \pi r^{2}(n)\left(1-\left(\pi r^{2}(n)+S_{i, j}^{\lambda_{b}}\right)\right)^{n^{d}} \\
& \quad+\left(1-4 \pi r^{2}(n)\right)\left(1-\left(2 \pi r^{2}(n)+S_{i, j}^{\lambda_{b}}\right)\right)^{n^{d}},
\end{aligned}
$$

where $S_{i, j}^{\lambda_{b}}$ is the total area covered by $i$ and $j$ during period $\lambda_{b}$. The two terms on the right hand side of (3) take into account the cases where the two nodes carrying the packet of $s_{i}$ and $s_{j}$ are at a distance less than $2 r(n)$ and greater than $2 r(n)$ when initially distributed, respectively.

Then we evaluate $S_{i, j}^{\lambda_{b}}$ as follows. We first study the area covered by movement during $\mathrm{k}$ time-slots of one node, i.e., $S_{i}^{\lambda_{b}}$. When a node is moving, the area covered in each timeslot may overlap due to the change of direction or the intersection of segments of the track, as depicted in Figure 4. Denote the two kinds of overlapped area by $S_{o}^{1}$ and $S_{o}^{2}$, respectively, and then considering the symmetry we have

$$
\begin{aligned}
S_{o}^{1} & =k \cdot \frac{1}{2} \int_{\varepsilon}^{\frac{\pi}{2}} 2 \frac{r(n)}{\sin \varphi} \cdot 2 r(n) \cdot \frac{d \varphi}{\pi / 2} \\
& =\frac{4 k r^{2}(n)}{\pi} \int_{\varepsilon}^{\frac{\pi}{2}} \frac{1}{\sin \varphi} \\
& =\left.\frac{4 k r^{2}(n)}{\pi} \cdot \frac{1}{2} \ln \frac{1+\cos \varphi}{1-\cos \varphi}\right|_{\varepsilon} ^{\frac{\pi}{2}} \\
& =o(r(n)), \text { as } n \rightarrow \infty .
\end{aligned}
$$
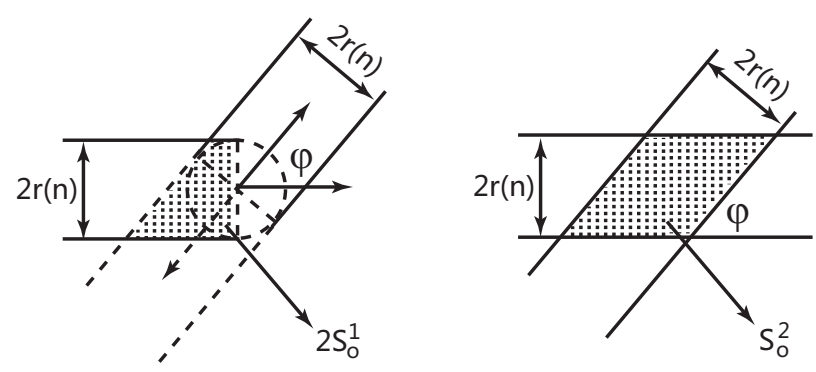

Figure 4: Overlapped area and

$$
\begin{aligned}
S_{o}^{2} & =l \cdot \int_{\varepsilon}^{\frac{\pi}{2}} \frac{2 r(n)}{\sin \varphi} \cdot 2 r(n) \cdot \frac{d \varphi}{\pi / 2}, \text { where } 0 \leq l \leq C_{k}^{2} \\
& =\left.\frac{8 l r^{2}(n)}{\pi} \cdot \frac{1}{2} \ln \frac{1+\cos \varphi}{1-\cos \varphi}\right|_{\varepsilon} ^{\frac{\pi}{2}} \\
& =o(r(n)), \text { as } n \rightarrow \infty .
\end{aligned}
$$

where $\varepsilon>0$, since the measure of $\varphi=0$ or $\pi$ is zero. Then we obtain

$$
\begin{aligned}
S_{i}^{\lambda_{b}} & \sim 2 r(n) k v T-S_{o}^{1}-S_{o}^{2} \\
& \sim 2 r(n) k v .
\end{aligned}
$$

Since the tracks of $i$ and $j$ are independent, the overlapped area of $S_{i}^{\lambda_{b}}$ and $S_{j}^{\lambda_{b}}$ can be also shown to be of order $o(r(n))$, therefore we have

$$
\begin{aligned}
S_{i, j}^{\lambda_{b}} & \sim S_{i}^{\lambda_{b}}+S_{j}^{\lambda_{b}} \\
& \sim 4 r(n) k v
\end{aligned}
$$

and therefore,

$$
\begin{aligned}
& P\left(\left\{s_{i} \text { and } s_{j} \text { are failed sessions in } \mathcal{G}_{r w} \text { in period } \lambda_{b}\right\}\right) \\
& \quad \leq(1-(1+\epsilon) 4 r(n) k v)^{n^{d}},
\end{aligned}
$$

for all $n>N(\epsilon)$ and $\epsilon=o(r(n))$.

To evaluate the left hand side of (4), we could directly apply the inequality

$$
1-x \leq e^{x} \text { for } x \in[0,1] .
$$

Here we introduce another approach which would benefit our other proofs. We first take the logarithm of the right hand side of (4), and we get

$$
\begin{aligned}
& \log (\text { R.H.S. of }(4)) \\
& =n^{d} \log (1-(1+\epsilon) 4 r(n) k v) \\
& =-n^{d} \sum_{i=1}^{\infty} \frac{((1+\epsilon) 4 r(n) k v)^{i}}{i} \\
& =-n^{d} \sum_{i=1}^{\infty}\left(\frac{1}{i}\left(2(1+\epsilon) \frac{\log n+\omega}{n^{d}}\right)^{i}\right) \\
& \leq-n^{d}\left(\sum_{i=1}^{2} \frac{1}{i}\left(2(1+\epsilon) \frac{\log n+\omega}{n^{d}}\right)^{i}+\delta(n)\right)
\end{aligned}
$$

where

$$
\begin{aligned}
\delta(n) & =\sum_{i=3}^{\infty} \frac{1}{i}\left(2(1+\epsilon) \frac{\log n+\omega}{n^{d}}\right)^{i} \\
& \geq \sum_{i=3}^{\infty}(1+\epsilon)^{i}\left(\frac{\log n+\omega}{n^{d}}\right)^{i} \\
& =(1+\epsilon)^{3}\left(\frac{\log n+\omega}{n^{d}}\right)^{3} /\left(1-(1+\epsilon) \frac{\log n+\omega}{n^{d}}\right) \\
& \geq(1+\epsilon)^{3}\left(\frac{\log n+\omega}{n^{d}}\right)^{3} .
\end{aligned}
$$


Substituting (7) in (6), we get

$$
\begin{aligned}
& \log (\text { R.H.S. of (4)) } \\
& \leq-n^{d}\left(2(1+\epsilon) \frac{\log n+\omega}{n^{d}}+2(1+\epsilon)^{2}\left(\frac{\log n+\omega}{n^{d}}\right)^{2}\right. \\
& \left.+(1+\epsilon)^{3}\left(\frac{\log n+\omega}{n^{d}}\right)^{3}\right) .
\end{aligned}
$$

Considering $\epsilon=o(r(n))=o\left(\frac{\log n}{n^{d}}\right)$, for any fixed $\tilde{\epsilon}>0$, we have

$$
\log (\text { R.H.S. of }(4)) \leq-2(1+\epsilon)(\log n+\omega)-\tilde{\epsilon},
$$

for all sufficiently large $n$. Thus,

$$
\begin{aligned}
& P\left(\left\{s_{i} \text { and } s_{j} \text { are failed sessions in } \mathcal{G}_{r w} \text { in period } \lambda_{b}\right\}\right) \\
& \quad \leq \theta e^{-2(1+\epsilon)(\log n+\omega)},
\end{aligned}
$$

where $\theta=e^{-\tilde{\epsilon}}$. Using (2) and (8) in (1), we obtain

$$
\begin{aligned}
P_{f_{-} r w}(n, r(n)) \geq & n(1-(1+\epsilon) 2 k v r(n))^{n^{d}} \\
& -\theta n^{2} e^{-2(1+\epsilon)(\log n+\omega)} .
\end{aligned}
$$

As to the first term $n f(n)$ on the right hand side of (9), where $f(n)=(1-(1+\epsilon) 2 k v r(n))^{n^{d}}$, recalling that $\epsilon=$ $o\left(\frac{\log n}{n^{d}}\right)$ we have

$$
\begin{aligned}
\log f(n) & =n^{d} \log (1-(1+\epsilon) 2 k v r(n)) \\
& \sim-2 k v n^{d} r(n)=-\log n-\omega .
\end{aligned}
$$

Therefore, $n f(n)=\Theta\left(n e^{-\log n-\omega}\right) \geq \theta^{\prime} e^{-\omega}$, for all $n \geq$ $N_{0}\left(\theta^{\prime}\right)$, where $\theta^{\prime}$ is a fixed positive constant. Actually, with more skilled technique, we can prove that $\theta^{\prime}<1$. $^{3}$

Using the above result, for any fixed $0<\theta<1$ and $0<$ $\theta^{\prime}<1$, we obtain

$$
P_{f_{-} r w}(n, r(n)) \geq \theta^{\prime} e^{-\omega}-\theta n^{2} e^{-2(1+\epsilon)(\log n+\omega)}
$$

for all $n>N\left(\epsilon, \theta, \theta^{\prime}, \omega\right)$.

Now we consider the case where $\omega$ is a function $\omega(n)$ with $\lim _{n \rightarrow \infty} \omega(n)=\hat{\omega}$. Then for all $n \geq \tilde{N}(\tilde{\epsilon})$ and any $\tilde{\epsilon}>0$, $\omega(n) \leq \hat{\omega}+\tilde{\epsilon}$. Considering that the probability of disconnectedness is monotonely decreasing in $\omega$ and taking limits, then we have

$$
\liminf _{n \rightarrow \infty} P_{f_{-} r w}(n, r(n)) \geq \theta^{\prime} e^{-(\hat{\omega}+\tilde{\epsilon})}-\theta e^{-2(\hat{\omega}+\tilde{\epsilon})} .
$$

Since this holds for all $\tilde{\epsilon}>0, \theta<1$ and $\theta^{\prime}<1$, the result follows.

As an obvious consequence of the Proposition 4.1, we have

COROLlary 4.1. Under the random walk mobility assumption, the mobile $k$-hop clustered network is to have failed sessions with positive probability bounded away from zero if $r(n)=\frac{\log n+\omega(n)}{2 k v n^{d}}$ and $\lim _{n \rightarrow \infty} \omega(n)<+\infty$.

Hence we have proved the necessity part of Theorem 4.1.

\footnotetext{
${ }^{3}$ Refer to the proof of Lemma 5.1 .
}

\subsection{Sufficient condition on $r(n)$ of Theorem 4.1}

Suppose there are at most $n$ sessions in a period $\lambda_{b}$, and let $E_{i}$ denote the event that $s_{i}$ is a failed session, where $i=1,2, \ldots, n$. Let each node have the transmission range $r=\operatorname{cr}(n)$, where $c>1$. Then it suffices to show that

$$
\lim _{n \rightarrow \infty} P\left(\bigcup_{i=1}^{n} E_{i}\right)=0 .
$$

Using the union bound we have

$$
P\left(\bigcup_{i=1}^{n} E_{i}\right) \leq \sum_{i=1}^{n} P\left(E_{i}\right) .
$$

As to each term in the summation on the right hand side of (10), we have

$$
P\left(E_{i}\right)=\left(1-\left(\pi r^{2}+S_{i}^{\lambda_{b}}\right)\right)^{n^{d}},
$$

where $S_{i}^{\lambda_{b}}$ is the area covered by movement during k timeslots of node $i$. Then applying the similar technique in the proof of necessity, we can show that

$$
P\left(E_{i}\right) \leq \theta \cdot \frac{1}{n^{c}}
$$

where $0<\theta<1$.

Thus, using (11) in (10), we have

$$
P\left(\bigcup_{i=1}^{n} E_{i}\right) \leq \frac{\theta}{n^{c-1}} .
$$

For any $c>1$, the result follows.

\section{THE CRITICAL TRANSMISSION RANGE FOR MOBILE K-HOP CLUSTERED NET- WORKS, I.I.D. MOBILITY}

The main result of this section is as follows.

TheOREM 5.1. Under the i.i.d. mobility assumption, the critical transmission range is $r(n)=\sqrt{\frac{\log n}{k \pi n^{d}}}$, where $\frac{1}{k}<$ $d \leq 1$.

\subsection{Necessary condition on $r(n)$ of Theorem 5.1}

We start with the following technical lemma.

LEMMA 5.1. If $\pi r^{2}(n)=\frac{\log n+\omega}{k n^{d}}$, for any fixed $\theta<1$ and $\mu \leq 1$, and for all sufficiently large $n$

$$
n\left(1-\mu \pi r^{2}(n)\right)^{k n^{d}} \geq \theta e^{-\omega},
$$

where $0<d \leq 1$.

Proof. Taking the logarithm of the left hand side of (12), we get

$$
\log (\text { L.H.S. of }(12))=\log n+k n^{d} \log \left(1-\mu \pi r^{2}(n)\right) .
$$

Using the power series expansion for $\log (1-x)$,

$$
\begin{aligned}
& \log (\text { L.H.S. of (12)) } \\
& \quad=\log n-k n^{d} \sum_{i=1}^{\infty} \frac{\left(\mu \pi r^{2}(n)\right)^{i}}{i} \\
& \quad=\log n-k n^{d}\left(\sum_{i=1}^{2} \frac{1}{i}\left(\mu \frac{\log n+\omega}{k n^{d}}\right)^{i}+\delta(n)\right),
\end{aligned}
$$


where

$$
\begin{aligned}
\delta(n) & =\sum_{i=3}^{\infty} \frac{1}{i}\left(\mu \frac{\log n+\omega}{k n^{d}}\right)^{i} \\
& \leq \frac{1}{3} \int_{2}^{\infty}\left(\mu \frac{\log n+\omega}{k n^{d}}\right)^{x} d x \\
& =\left.\frac{1}{3 \log \left(\mu \frac{\log n+\omega}{k n^{d}}\right)}\left(\mu \frac{\log n+\omega}{k n^{d}}\right)^{x}\right|_{2} ^{\infty} \\
& \leq \frac{1}{3}\left(\mu \frac{\log n+\omega}{k n^{d}}\right)^{2}
\end{aligned}
$$

for all large $n$. Substituting (15) in (14), we get

$$
\begin{aligned}
& \log (\text { L.H.S. of (12)) } \\
& \quad \geq \log n-k n^{d}\left(\mu \frac{\log n+\omega}{k n^{d}}+\frac{5}{6}\left(\mu \frac{\log n+\omega}{k n^{d}}\right)^{2}\right) \\
& \quad=(1-\mu) \log n-\mu \omega-\frac{5 \mu^{2}(\log n+\omega)^{2}}{6 k^{2} n^{2 d}} \\
& \quad \geq-\omega-\epsilon,
\end{aligned}
$$

for all sufficiently large $n$. Taking the exponent of both sides and using $\theta=e^{-\epsilon}$, the result follows.

Let $\mathcal{G}_{\text {iid }}(n, r(n))$ denote the network where two nodes can communicate if their Euclidean distance is at most $r(n)$ and $P_{f_{-i i d}}(n, r(n))$ be the probability that $\mathcal{G}_{i i d}(n, r(n))$ has failed sessions (i.e., it has a node that is not connected). Then we have the following proposition.

Proposition 5.1. If $\pi r^{2}(n)=\frac{\log n+\omega(n)}{k n^{d}}$, where $\frac{1}{k}<d \leq$ 1 , then

$$
\liminf _{n \rightarrow \infty} P_{f_{-i i d}}(n, r(n)) \geq e^{-\omega}\left(1-e^{-\omega}\right),
$$

where $\omega=\lim _{n \rightarrow \infty} \omega(n)$.

Proof. The proof applies similar techniques as that of Proposition 4.1, and we only illustrate several critical steps while others are omitted for the sake of brevity.

To evaluate $P_{f_{-} i i d}(n, r(n)$, we have

$$
\begin{gathered}
P_{f_{-i i d}}(n, r(n)) \\
\geq \sum_{i=1}^{n} P\left(\left\{s_{i} \text { is a failed session in } \mathcal{G}_{i i d}(n, r(n))\right\}\right) \\
-\sum_{i=1}^{n} \sum_{j \neq i} P\left(\left\{s_{i} \text { and } s_{j}\right.\right. \text { are failed sessions } \\
\left.\left.\quad \text { in } \mathcal{G}_{i i d}(n, r(n))\right\}\right) .
\end{gathered}
$$

Then we evaluate the two terms on the right hand side of (16), respectively, and we have

$$
P\left(\left\{s_{i} \text { is a failed session }\right\}\right) \geq\left(\left(1-\pi r^{2}(n)\right)^{n^{d}}\right)^{k}
$$

and

$$
\begin{aligned}
& P\left(\left\{s_{i} \text { and } s_{j} \text { are failed sessions }\right\}\right) \\
& \leq\left(4 \pi r^{2}(n)\left(1-\pi r^{2}(n)\right)^{n^{d}}\right. \\
& \left.\quad+\left(1-4 \pi r^{2}(n)\right)\left(1-2 \pi r^{2}(n)\right)^{n^{d}}\right)^{k} .
\end{aligned}
$$

Using (17) and (18) in (16), we obtain

$$
\begin{aligned}
& P_{f_{-} i i d}(n, r(n)) \\
& \geq n\left(1-\pi r^{2}(n)\right)^{k n^{d}}-n^{2}\left(4 \pi r^{2}(n)\left(1-\pi r^{2}(n)\right)^{n^{d}}\right. \\
& \left.+\left(1-2 \pi r^{2}(n)\right)^{n^{d}}\right)^{k} .
\end{aligned}
$$

Using Lemma 5.1 and (5), for any fixed $\theta<1$, we have

$$
\begin{aligned}
& P_{f_{-} i i d}(n, r(n)) \\
& \geq \theta e^{-\omega}-\left(n^{\frac{2}{k}}\left(4 \pi r^{2}(n) e^{-n^{d} \pi r^{2}(n)}+e^{-2 n^{d} \pi r^{2}(n)}\right)\right)^{k} \\
& =\theta e^{-\omega}-\left(\frac{4(\log n+\omega)}{k n^{d-\frac{1}{k}}} e^{-\frac{1}{k} \omega}+e^{-\frac{2}{k} \omega}\right)^{k} \\
& \geq \theta e^{-\omega}-(1+\epsilon) e^{-2 \omega}
\end{aligned}
$$

for any $\epsilon>0$ and for all $n>N(\epsilon, \theta, \omega)$.

Now let $\omega$ be a function $\omega(n)$ with $\lim _{n \rightarrow \infty} \omega(n)=\hat{\omega}$. Then for all $n \geq N^{\prime}(\epsilon)$ and any $\epsilon>0, \omega(n) \leq \hat{\omega}+\epsilon$. Considering that the probability of disconnectedness is monotonely decreasing in $\omega$, then we have

$$
P_{f_{-} i \mathrm{id}}(n, r(n)) \geq \theta e^{-(\hat{\omega}+\epsilon)}-(1+\epsilon) e^{-2(\hat{\omega}+\epsilon)},
$$

for $n \geq \max \left\{N(\epsilon, \theta, \hat{\omega}+\epsilon), N^{\prime}(\epsilon)\right\}$. Taking limits

$$
\liminf _{n \rightarrow \infty} P_{f_{-i i d}}(n, r(n)) \geq \theta e^{-(\hat{\omega}+\epsilon)}-(1+\epsilon) e^{-2(\hat{\omega}+\epsilon)} .
$$

Since this holds for all $\epsilon>0$ and $\theta<1$, the result follows.

Then we have the following corollary to prove the necessity part of Theorem 6.1.

COROLlary 5.1. Under the i.i.d. mobility assumption, the mobile $k$-hop clustered network is to have failed sessions with positive probability bounded away from zero if $\pi r^{2}(n)=$ $\frac{\log n+\omega(n)}{k n^{d}}$ and $\lim _{n \rightarrow \infty} \omega(n)<+\infty$.

\subsection{Sufficient condition on $r(n)$ of Theorem 5.1}

Suppose there are at most $n$ sessions in a period $\lambda_{b}$, and let $E_{i}$ denote the event that $s_{i}$ is a failed session, where $i=1,2, \ldots, n$. Let each node have the transmission range $r=\operatorname{cr}(n)$, where $c>1$. Using the existing approach in Section 4.2 , we have

$$
\begin{aligned}
P\left(\bigcup_{i=1}^{n} E_{i}\right) & \leq \sum_{i=1}^{n} P\left(E_{i}\right) \\
& \leq n\left(\left(1-\pi r^{2}\right)^{n^{d}}\right)^{k} \\
& \leq n e^{-k n^{d} \pi r^{2}} \\
& =\frac{1}{4 n^{c^{2}-1}}
\end{aligned}
$$

For any $c>1$, the result follows. 


\section{THE CRITICAL TRANSMISSION RANGE FOR CONNECTIVITY OF STATIONARY K-HOP CLUSTERED NETWORKS}

In this section, our main result is the following theorem.

THEOREM 6.1. For the stationary $k$-hop clustered networks, the critical transmission range is $r(n)=\frac{1}{k} \sqrt{\frac{d \log n}{\pi n^{d}}}$, where $0<d<1$.

\subsection{Necessary condition on $r(n)$ of Theorem 6.1}

Let $\mathcal{G}_{\text {stat }}(n, r(n))$ denote the network where two nodes are connected if their Euclidean distance is at most $r(n)$ and we use the term disconnected to describe a cluster member whose packets cannot reach a cluster head within $k$ hops. Then, if there is at least one disconnected member node in the network, we define that $\mathcal{G}_{\text {stat }}$ is disconnected. Let $P_{d_{\text {sstat }}}(n, r(n))$ be the probability that $\mathcal{G}_{\text {stat }}$ is disconnected and we have the following proposition. then

Proposition 6.1. If $\pi r^{2}(n)=\frac{d_{0} \log n+\omega}{k^{2} n^{d}}$, where $d_{0}<d$,

$$
\liminf _{n \rightarrow \infty} P_{d_{-} \text {stat }}(n, r(n)) \geq e^{-\omega}\left(1-e^{-\omega}\right),
$$

where $\omega=\lim _{n \rightarrow \infty} \omega(n)$.

Proof. It can be shown that we cannot develop the necessary condition on $r(n)$ using the previous approach when $d$ is strictly less than 1 . In that approach, we considered all pairs of nodes $i$ and $j$ for the event that

$\{i$ and $j$ are disconnected cluster members $\}$.

The problem of the above approach is that there are too many of these pairs. The intuitive explanation is depicted in the following figure.

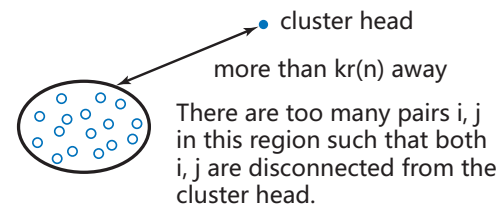

Figure 5: An intuitive explanation of the problem in the above approach

Further, such $i$ and $j$ may be very close to each other, making it difficult to bound the probability.

Instead, in the following we only consider a selected set of $i$ s and $j$ s. Let $u(n)=O\left(\sqrt{\frac{\log n}{n}}\right)$. Then we divide the unit square into $\frac{1}{u(n)} \times \frac{1}{u(n)}$ cells such that each cell is of size $u^{2}(n)$.

Now, among these cells, pick $n^{d_{0}}$ of them such that each of them is at least $\sqrt{\frac{1}{n^{d}}}$ away from others, as shown in Figure 6.

Note that by construction, these selected cells form a grid. Pick one cluster member node from each of these selected cells. There are a total of $n^{d_{0}}$ of these nodes. Let $Y$ denote the set of such cluster member nodes. Note that any two nodes in $Y$ are at a distance of at least $\sqrt{\frac{1}{n^{d_{0}}}}$ away. Further, this distance is larger than $k r(n)$ when $n$ is sufficiently large.

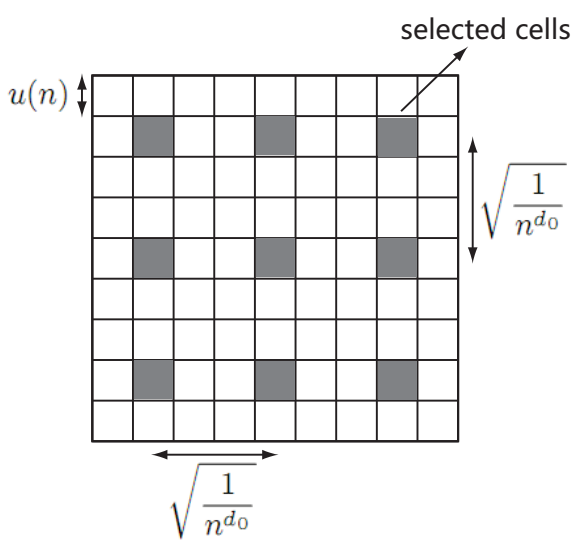

Figure 6: Cell selection

Then, using the existing approach of computing the probability that the network is disconnected, we have

$$
\begin{aligned}
& P_{d \_ \text {stat }}(n, r(n)) \\
& \geq \sum_{i \in Y} P(\{i \text { is an disconnected cluster member } \\
& \left.\left.\quad \text { in } \mathcal{G}_{\text {stat }}(n, r(n))\right\}\right) \\
& \quad-\sum_{\substack{i, j \in Y, j \neq i}} P(\{i \text { and } j \text { are disconnected cluster }
\end{aligned}
$$$$
\text { members in } \left.\left.\mathcal{G}_{\text {stat }}(n, r(n))\right\}\right) \text {. }
$$

As to the first term in the right hand side of (20), it is bounded by

$$
n^{d_{0}}\left(1-\pi(k r(n))^{2}\right)^{n^{d}} \geq n^{d_{0}} \cdot \theta \cdot \frac{1}{n^{d_{0}}} e^{-\omega}=\theta e^{-\omega},
$$

where $0<\theta<1$. To bound the second term, note that $i, j \in Y$ are at least $\sqrt{\frac{1}{n^{d_{0}}}}$ away, which will be larger than $2 k r(n)$ when $n$ is sufficiently large. Thus, each term in the second summation is bounded by

$$
P(\{i \text { and } j \text { are disconnected }\}) \leq\left(1-2 \pi(k r(n))^{2}\right)^{n^{d}} .
$$

Therefore,

$$
\begin{aligned}
P_{d_{-} s t a t}(n, r(n)) & \geq \theta e^{-\omega}-n^{2 d_{0}} e^{-2 n^{d} \pi(k r(n))^{2}} \\
& \geq \theta e^{-\omega}-e^{-2 \omega},
\end{aligned}
$$

for all $n>N(\theta, \omega)$.

The rest of steps are omitted and follows these in the earlier proofs.

Remark 6.1. Unlike Proposition 4.1 or 5.1, Proposition 6.1 provides the necessary condition on $r(n)$ in terms of both $\omega(n)$ and $d_{0}$, i.e., in addition to the requirement for $\omega(n)$ to approach infinity, this condition also says that any $d_{0}<d$ will result in a positive probability of disconnection. To explain this implication, suppose two range $r_{0}(n)$ and $r_{1}(n)$, such that $\pi r_{0}^{2}(n)=\frac{d_{0} \log n+\omega(n)}{k^{2} n^{d}}$ and $\pi r_{1}^{2}(n)=\frac{d_{1} \log n+\omega}{k^{2} n^{d}}$, where $d_{0}<d_{1}<d, \omega(n) \stackrel{k^{2} n^{d}}{\rightarrow} \infty$ and $\omega$ is a constant. Note that we actually assumed $\omega(n)=o(\log n)$ in related proofs. By Proposition 6.1, we know that $r_{1}(n)$ will result in a disconnected network. Meanwhile, we have $r_{0}(n)<r_{1}(n)$ for all sufficiently large $n$ and the probability of disconnection is 
monotonely decreasing in $r$. Therefore, $r_{0}(n)$ will also result in a disconnected network.

Referring to Remark 6.1, we have the following corollary.

Corollary 6.1. $r(n) \geq \frac{1}{k} \sqrt{\frac{d \log n}{\pi n^{d}}}$ is necessary for the connectivity of stationary $k$-hop clustered networks.

\subsection{Sufficient condition on $r(n)$ of Theorem 6.1}

Heuristically, we can use the tessellation-based approach to prove the sufficiency part of Theorem 6.1. However, it can also be shown that we cannot develop a sufficient condition on $r(n)$ by directly applying the similar technique as in the previous proofs. The problem is that when $\frac{1}{k}<d \leq 1$, we cannot bound the probability that at least one cell has no cluster heads to asymptotically approach zero, if the size of cell is roughly smaller than $\pi(k r(n))^{2}$. On the other side, when the unit square is tessellated such that the size of cell is roughly equal to $\pi(k r(n))^{2}$, we are also unable to show that any clustered head within the distance of $k r(n)$ is reachable for a cluster member. However, we can overcome this technical problem by the trick of considering a distance that is reachable for a cluster member and approaches $k r(n)$ as well.

First, we divide the unit square into cells with side length $\frac{\sqrt{2}}{2} \frac{r(n)}{\log n}$. Thus, there are $\log n$ cells along the transmission range $r(n)$. This tessellation is shown in Figure 7 .

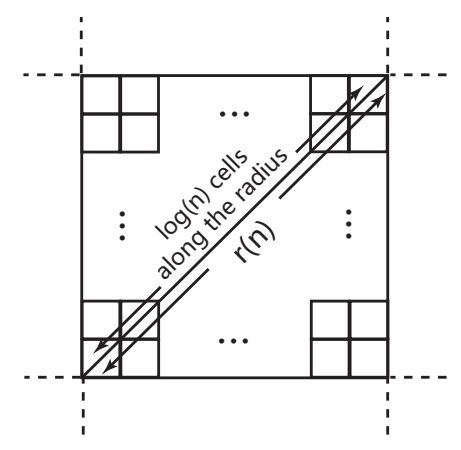

Figure 7: There are $\log n$ cells along the transmission range $r(n)$.

Then we evaluate the probability that at least one cell is empty, and we have

$$
\begin{aligned}
& P(\{\text { at least one cell is empty }\}) \\
& \quad \leq \frac{2}{d} \pi k^{2} n^{d} \log n\left(1-\frac{d}{2 \log n \pi k^{2} n^{d}}\right)^{n} \\
& \quad \leq \frac{2}{d} \pi k^{2} \log n / n^{\frac{d}{2 \pi k^{2}} \frac{n^{1-d}}{\log ^{2} n}-d} \\
& \quad \rightarrow 0, \text { as } n \rightarrow \infty
\end{aligned}
$$

Consequently, we know that with high probability there is at least one node in each cell. Then, with the transmission range $r(n)$, each hop can jump over at least $(\log n-1)$ cells. Thus, any cluster head within the distance of $\frac{\log n-1}{\log n} k r(n)$ is reachable in $k$ hops with high probability. Now we have a proper cell size to construct the main tessellation.

Next, we introduce the disk tessellation (with a minor abuse of the term tessellation) of the unit torus, as depicted in Figure 8. Let the radius of each disk $R(n)$ be such that
$R(n)=\frac{\log n-1}{\log n} k r(n)$. Then we evaluate the probability that at least one disk does not have cluster heads, and we have

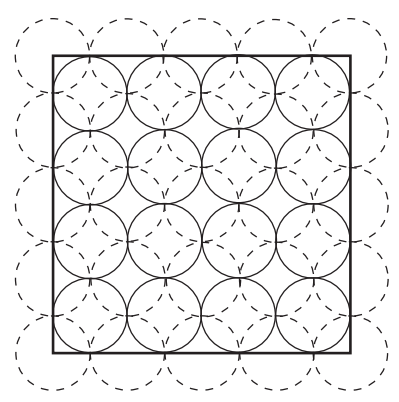

Figure 8: Disk tessellation of the unit torus

$$
\begin{aligned}
& P(\{\text { at least one disk does not have cluster heads }\}) \\
& \leq 2\left(\frac{1}{2\left(\frac{\log n-1}{\log n} k r(n)\right)}\right)^{2}\left(1-\pi\left(\frac{\log n-1}{\log n} k r(n)\right)^{2}\right)^{n^{d}} \\
& \leq \frac{\pi n^{d} \log n}{2 d(\log n-1)^{2}} \exp \left\{-\left(\frac{\log n-1}{\log n}\right)^{2} n^{d} \pi(k r(n))^{2}\right\} \\
& \leq \frac{\pi \log n}{2 d(\log n-1)^{2}} n^{d-d\left(\frac{\log n-1}{\log n}\right)^{2}} \\
& \rightarrow 0, \text { as } n \rightarrow \infty
\end{aligned}
$$

Therefore, $r(n)=\frac{1}{k} \sqrt{\frac{d \log n}{\pi n^{d}}}$ is sufficient to guarantee the connectivity of network.

\section{THE IMPACT OF MOBILITY ON CON- NECTIVITY AND NETWORK PERFOR- MANCE IN K-HOP CLUSTERED NET- WORKS}

In this section, we use the results that we obtained from the previous sections to study the impact of mobility on connectivity and network performance. We first characterize the power-delay trade-off and energy consumption per flow in clustered networks, based on the critical transmission ranges that we have obtained. We then summarize these results and explore the insights and implications that they may provide us.

We assume the free space propagation model ${ }^{4}$, and thus

$$
P_{r}=P_{t} G_{t} G_{r}\left(\frac{\lambda}{4 \pi d}\right)^{2}
$$

where

$$
\begin{aligned}
P_{t} & =\text { transmission power of an isotropic source, } \\
G_{t} & =\text { transmitting antenna gain, } \\
G_{r} & =\text { receiving antenna gain, } \\
d & =\text { propagation distance between antennas, } \\
\lambda & =\text { carrier wavelength. }
\end{aligned}
$$

${ }^{4}$ Other propagation model (e.g., the two-ray model) can be studied in a similar way. 


\begin{tabular}{|c|c|c|c|c|c|c|c|}
\hline \multicolumn{3}{|c|}{ Network Type } & $\begin{array}{l}\text { Transmission } \\
\text { Range }(r(n))\end{array}$ & $\begin{array}{l}\text { Transmission Power }\left(P_{t}\right) \\
\text { vs. Delay }(D) \text { Trade-off }\end{array}$ & $\begin{array}{c}\text { Average } \\
\text { Hops }(\bar{H})\end{array}$ & $\begin{array}{l}\text { Average Energy } \\
\text { Cons. per Flow }(\bar{E})\end{array}$ & $\begin{array}{l}\text { Cluster Head } \\
\text { Exponent }(d)\end{array}$ \\
\hline \multicolumn{3}{|c|}{ Flat Network } & $\sqrt{\frac{\log n}{\pi n}}$ & $P_{t}=\Theta\left(\frac{\log n}{n}\right)$ & $\Theta\left(\sqrt{\frac{n}{\log n}}\right)$ & $\Theta\left(\frac{\sqrt{\log n}}{n^{1 / 2}}\right)$ & \\
\hline \multirow{3}{*}{$\begin{array}{l}\text { Clustered } \\
\text { Network }\end{array}$} & \multicolumn{2}{|c|}{ Stationary } & $\frac{1}{k} \sqrt{\frac{d \log n}{\pi n^{d}}}$ & $P_{t}=\Theta\left(\frac{d}{D^{2}} \frac{\log n}{n^{d}}\right)$ & $\Theta(1)$ & $\Theta\left(\frac{\log n}{n^{d}}\right)$ & $0<d<1$ \\
\hline & \multirow{2}{*}{ Mobile } & $\begin{array}{l}\text { I.I.D. } \\
\text { Mobility }\end{array}$ & $\sqrt{\frac{\log n}{k \pi n^{d}}}$ & $P_{t}=\Theta\left(\frac{1}{D} \frac{\log n}{n^{d}}\right)$ & $\Theta(1)$ & $\Theta\left(\frac{\log n}{n^{d}}\right)$ & $\frac{1}{k}<d \leq 1$ \\
\hline & & $\begin{array}{c}\text { R.W. } \\
\text { Mobility } \\
\text { (Non-trivial) }\end{array}$ & $\frac{\log n}{2 k v n^{d}}$ & $P_{t}=\Theta\left(\frac{1}{D^{2}}\left(\frac{\log n}{n^{d}}\right)^{2}\right)$ & $\Theta(1)$ & $\frac{\log n}{n^{d}}$ & $0<d \leq 1$ \\
\hline
\end{tabular}

Table 1: The transmission power vs. delay trade-off and energy consumption in $k$-hop clustered networks

Let $G_{t}, G_{r}$ and $\lambda$ be constants. We then have

$$
P_{t}=\frac{1}{G_{t} G_{r}}\left(\frac{4 \pi d}{\lambda}\right)^{2} P_{r}=c P_{r} d^{2}
$$

where $c$ is a constant. To ensure sufficient signal strength for receiving the packet, we require that $P_{r} \geq P_{r_{-} t h}$, where $P_{r_{-} t h}$ is a threshold of the receiving power at the receiver. Replace $P_{r}$ with $P_{r_{-} t h}$ in (21) and replace the propagation distance $d$ by the transmission range $r$. We then have

$$
P_{t} \propto r^{2}
$$

Consequently, let $\bar{E}$ denote the energy consumption per flow. We have

$$
\bar{E}=\bar{H} P_{t} \propto \bar{H} r^{2},
$$

where $\bar{H}$ is the average number of hops per flow.

$P_{t}$ and $\bar{E}$ are both of great engineering significance, while they have different influence on a network. $P_{t}$ is correlated to the node-level operation and it has a dominating impact on the total number of transmissions that a single node can undertake in energy-constrained networks like wireless sensor networks [26]. On the other hand, $\bar{E}$ is a flow-level description of energy consumption and thus it provides a picture of the life-time expectation both of each single node and of the entire network.

Using the results in the critical transmission range $r(n)$ from the earlier sections, we can compute the order of $P_{t}$ and $\bar{E}$. All the results in this paper are reported in Table 1.

Before we discuss these results, we make a cautious note regarding the energy consumption. Note that in these calculations, we have ignored the energy consumption due to mobility. Hence, these results should not be interpreted as a reason to introduce mobility to an otherwise static network, but rather represent an inherent advantage of having mobility in the system. Similarly, the comparison with the flat network is not entirely fair, since in a clustered network, a packet only needs to reach a cluster head. Hence, our following results should be viewed as an inherent advantage of clustered network due to the availability of infrastructure support.

We now discuss the insights on the impact of mobility on connectivity and network performance based on these results. By the implication from [27], we know that when $d<\frac{1}{2}$, bottleneck of capacity may appear, and thus we assume $d>\frac{1}{2}$ in our following discussion.
- $P_{t}\{$ r.w. $\}=o\left(P_{t}\{\right.$ flat $\left.\}\right)$ and $\bar{E}\{$ r.w. $\}=o(\bar{E}\{$ flat $\})$ which means that random walk mobility with non-trivial velocity plus $k$-hop clustering can greatly decrease both the transmission power and the average energy consumption per flow. Thus, random walk mobility with non-trivial velocity plus $k$-hop clustering can increase the number of transmission that a node can undertake and extend the life-time both of each single node and of the entire network.

- To identify the contribution of mobility and $k$-hop clustering on the improvement of network performance, we have

$$
\bar{E}\{\text { r.w. }\}=\frac{\log n}{n^{d}} \bar{E}\{\text { stat }\} ; P_{t}\{\text { r.w. }\}=\frac{\log n}{n^{d}} P_{t}\{\text { stat }\},
$$

and

$$
\bar{E}\{\text { stat }\}=\frac{\sqrt{\log n}}{n^{d-1 / 2}} \bar{E}\{\text { flat }\} ; P_{t}\{\text { stat }\}=n^{1-d} P_{t}\{\text { flat }\} .
$$

Thus, combining the results of above equations and using $\Uparrow$ and $\Downarrow$ to denote the positive and negative impacts, respectively, we provide the following formulations to identify the effects of mobility and $k$-hop clustering on network performance.

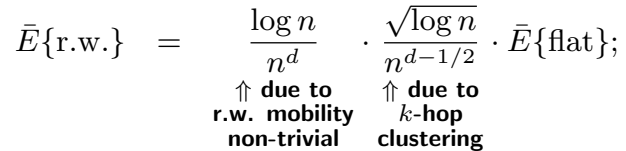

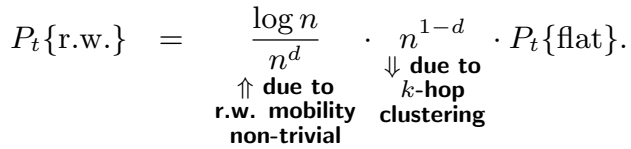

- From the perspective of energy consumption per flow, clustered networks have an inherent advantage in terms of energy-efficiency due to the availability of infrastructure support.

- Mobile $k$-hop clustered networks under the i.i.d mobility model and stationary clustered networks may have comparable performance and this can be understood intuitively since nodes under the i.i.d. mobility model actually remain static during the time-slot. 
In conclusion, random walk mobility with non-trivial velocity increases connectivity in $k$-hop clustered networks, and thus significantly improves the energy efficiency and the power-delay trade-off of the network.

\section{CONCLUDING REMARKS}

In this paper, we have studied the effects of mobility on the critical transmission range for asymptotic connectivity in $k$-hop clustered networks. Our contributions are twofold. We have developed the critical transmission range for the mobile $k$-hop clustered network under the random walk mobility model with non-trivial velocity and the i.i.d. mobility model, and for the stationary $k$-hop clustered network, respectively. These formulations do not only provide an asymptotic description of the critical power needed to maintain the connectivity of the network, but also help to identify the contribution of mobility in the improvement of network performance. Thus, based on these results that we have developed in this paper, our second contribution is to present that random walk mobility with non-trivial velocity increases connectivity in $k$-hop clustered networks, and thus significantly improves the energy efficiency and the powerdelay trade-off of the network.

There are several interesting directions for future work. First, we plan to extend the results for the random walk mobility model to account for the case where each node moves with different speed. For example, the node velocity may be randomly chosen between a minimum value and a maximum value, and the gap between these two values can be a function of $n$. Second, in our current model for random walk, each node changes direction after one time-slot. An interesting extension is to study the case where the change of directions occurs at random times (e.g., a node may move a random distance before it changes direction). In that case, given a delay constraint, the number of trips that a node can traverse before the packet expires will also become a random variable. It would be interesting to study whether such randomness can affect the connectivity results in this paper. Third, we plan to extend the results to account for wireless interference in the system. Finally, in this paper we assume that the cluster heads are stationary, even though the cluster members may move. It would be interesting to study the case where cluster-heads may move as well.

\section{ACKNOWLEDGMENT}

The authors thank Microsoft Research Asia for the helpful discussions during the authors visit MSRA. This work is supported by NSF China (No. 60702046, 60832005); China Ministry of Education (No. 20070248095); Shanghai Jiaotong University Young Faculty Funding (No. 06ZBX800050); Qualcomm Research Grant; China International Science and Technology Cooperation Programm (No. 2008DFA11630); PUJIANG Talents (08PJ14067); Shanghai Innovation Key Project (08511500400), and has been partially supported by the National Science Foundation through award CNS0721477 .

\section{REFERENCES}

[1] P. Gupta and P.R. Kumar, "Critical Power for Asymptotic Connectivity in Wireless Networks," Stochastic Analysis, Control, Optimization and
Applications: A Volume in Honor of W.H. Fleming, W.M. McEneaney, G. Yin, and Q. Zhang, Boston: Birkhauser, 1998.

[2] M.D. Penrose, "The Longest Edge of the Random Minimal Spanning Tree," Annals of Applied Probability, vol. 7, pp. 340-361, 1997.

[3] P. Wan and C.W. Yi, "Asymtotic Critical Transmission Radius and Critical Neighbor Number for K-connectivity in Wireless Ad Hoc Networks," in Proc. ACM MobiHoc 2004, Roppongi, Japan, May 24-26, 2004.

[4] F. Xue and P.R. Kumar, "The Number of Neighbors Needed for Connectivity of Wireless Networks," Wireless Networks, vol. 10, no. 2, pp. 169-181, 2004.

[5] R.Wattenhofer, L. Li, P. Bahl and Y.M. Wang, "Distributed Topology Control for Power Efficient Operation in Multi-hop Wireless Ad Hoc Networks," in Proc. IEEE INFOCOM 2001, pp. 1388-1397, Anchorage, AK, USA, April 2001.

[6] L. Li, J.Y. Halpern, V. Bahl, Y.M. Wang and R. Wattenhofer, "Analysis of A Cone-based Distributed Topology Control Algorithm for Wireless Multi-hop Networks," in Proc. ACM Symposium on Principles of Distributed Computing (PODC), pp. 264-273, Newport, RI, August 2001.

[7] F. Xue and P.R. Kumar, "On the $\theta$-coverage and Connectivity of Large Random Networks," the joint special issue of the IEEE Trans. on Information Theory and the IEEE/ACM Transactions on Networking on "Networking and Information Theory", May 2006.

[8] P. Gupta and P.R. Kumar, "The Capacity of Wireless Networks," IEEE Transactions on Information Theory, vol. 46, pp. 388-404, March 2000.

[9] P. Gupta, R. Gray, and P.R. Kumar, "An Experimental Scaling Law for Ad Hoc Networks," Univ. of Illinois at Urbana-Champaign, May 2001.

[10] W. Heinzelman, A. Chandrakasan and H. Balakrishnan, "Energy-efficient Communication Protocol for Wireless Micro Sensor Networks," in Proc. the 33rd Annual Hawaii International Conference on System Sciences, pp. 3005-3014, 2000.

[11] Qiangfeng Jiang and D. Manivannan, "Routing Protocols for Sensor Networks," in Consumer Communications and Networking Conference (CCNC 2004), pp. 93-98, 2004.

[12] V. Kawadia and P. R. Kumar, "Power Control and Clustering in Ad Hoc Networks," in Proc. IEEE INFOCOM 2003, vol. 1, pp. 459-469, San Francisco, CA, USA, March 30-April 3, 2003.

[13] A.D. Amis, R. Prakash, T.H.P. Vuong and D.T. Huynh, "Max-Min D-Cluster Formation in Wireless Ad Hoc Networks," in Proceedings of INFOCOM 2000, vol. 1, pp. 32-41, Tel Aviv, Israel, 2000.

[14] Y. Fernandess and D. Malkhi, "K-clustering in Wireless Ad Hoc Networks," in Proc. the Second ACM international Workshop on Principles of Mobile Computing, pp. 31-37, Toulouse, France, October 30-31, 2002.

[15] S. Bandyopadhyay and E.J. Coyle, "An Energy Efficient Hierarchical Clustering Algorithm for Wireless Sensor Networks," in Proc. IEEE INFOCOM 
2003, vol. 3, pp. 1713-1723, San Francisco, CA, USA, March 30-April 3, 2003.

[16] M. Younis, M. Youssef, and K. Arisha, "Energy-aware Routing in Cluster- based Sensor Networks," in Proc. IEEE International Symposium on Modeling, Analysis and Simulation Computer and Telecommunications Systems, pp. 129-136, 2002.

[17] M. Bechler, H.J. Hofi, D. Kraft, F. Pählke and L. Wolf, "A Cluster-based Security Architecture for Ad Hoc Networks," in Proc. IEEE INFOCOM 2004, vol. 4, pp. 2393-2403, Hong Kong, China, Mar 2004.

[18] M. Chatterjee, S.K. Das and D. Turgut, "WCA: $A$ Weighted Clustering Algorithm for Mobile Ad hoc networks," Cluster Computing, vol. 5, no. 2, pp. 193-204, April 2002.

[19] U. Kozat and L. Tassiulas, "Throughput capacity of random ad hoc networks with infrastructure support," in Proc. ACM MobiCom 2003, Annapolis, MD, USA, June 2003.

[20] M. Grossglauser and D. Tse, "Mobility Increases the Capacity of Ad Hoc Wireless Networks," IEEE/ACM Transactions on Networking, vol. 10, no. 4, pp. 477-486, August 2002.

[21] S. Čapkun, J. Hubaux and L. Buttyán, "Mobility Helps Security in Ad Hoc Networks," in Proc. ACM MobiHoc 2003, June 2003.

[22] T. Camp, J. Boleng and V. Davies, "A Survey of Mobility Models for Ad Hoc Network Research," Wireless Communication \& Mobile Computing (WCMC): Special issue on Mobile Ad Hoc Networking: Research, Trends and Applications, vol. 2, no. 5, pp. 483-502, 2002.

[23] Shu Du, David B. Johnson, and Amit Kumar Saha, "RMAC: A Routing-Enhanced Duty-Cycle MAC Protocol for Wireless Sensor Networks," in Proc. IEEE INFOCOM 2007, pp. 1478-1486, Anchorage, AK, USA, May 2007.

[24] Yanjun Sun, Shu Du, Omer Gurewitz, and David B. Johnson, "DW-MAC: A Low Latency, Energy Efficient Demand-Wakeup MAC Protocol for Wireless Sensor Networks," in Proc. ACM MobiHoc 2008, pp. 53-62, ACM, Hong Kong SAR, China, May 2008.

[25] R. B. Ash and C. A. Doléans-Dade, "Probability and Measure Theory," US: Academic Press, 2nd edition, 2000.

[26] I.F. Akyildiz, W. Su, Y. Sankarasubramaniam and E. Cayirci, "Wireless Sensor Networks: A Survey," Computer Networks, vol. 38, pp. 393-422, 2002.

[27] S. Toumpis, "Capacity Bounds for Three Classes of Wireless Networks: Asymmetric, Cluster, and Hybrid," in Proc. ACM MobHoc 2004, pp. 133-144, Roppongi, Japan, May 24-26, 2004. 